\title{
缺陷 LB 膜诱导草酸钙晶体环图形形成
}

\author{
欧阳健明* 邓穗平 \\ (暨南大学生物矿化与结石病防治研究所, 广州 510632)
}

\begin{abstract}
摘要利用存在缺陷的 LB 膜来模拟受损伤的肾上皮细胞膜, 研究了膜缺陷的微结构及其影响 一水草酸钙(COM) 晶体成核、生长、沉积图形和黏附的分子机制. 二棕榈酰磷脂酰胆碱(DPPC)LB 膜经草酸钾处理后可形成圆形缺陷, 并诱导 COM 微晶排列成环状图形, 相比之下, 没有经草酸 钾处理的 LB 膜只诱导零散的六边形 COM 晶体生成. 随着生长时间增加, 环内单个 COM 晶体的 尺寸明显增加, 空心的环状晶体图形倾向于转变为实心的圆形图形, 且小尺寸 $(5 \sim 20 \mu \mathrm{m})$ 的圆形 晶体图案数量增加. 本结果有助于在分子和超分子的水平上进一步了解肾上皮细胞膜损伤后诱 导肾结石形成的分子机制.
\end{abstract}

\section{关键词缺陷 LB 膜 草酸钙 SEM 生物矿化}

从物理化学的观点看, 肾结石形成至少与 3 个因 素密切相关 [1]: (1) 尿液中尿石盐的高度过饱和; (2) 抑制剂浓度或活性降低, 促进剂浓度增加; (3) 肾上 皮细胞膜表面损伤. 从分子和超分子的水平上, 肾上 皮细胞膜表面损伤后, 膜的极性、磷脂的对称性和有 序分子阵列遭到破坏, 暴露出更多的晶体黏附位点, 从而促进晶体的黏附和滞留 ${ }^{[2 \sim 4]}$.

Langmuir-Boldgett(LB) 膜的缺陷是限制其作为 一种纳米级材料应用的主要原因之一 [5]. LB膜的缺 陷小到分子级别的晶格位错、针孔、微畴区内分子排 列的有序性与微畴区边缘分子的无序性差异等, 大 到成膜分子所形成的微晶、台阶、层与层之间的大规 模重排 $[6 \sim 8]$, 这种重排是由于LB膜从堆积结构到热力 学稳定结构的转变; 此外, 还有膜崩溃而形成的堆积 /多层膜. 但另一方面, 由于LB膜具有与细胞膜类似 的结构, 因此, 可将LB膜作为模拟细胞膜的模型, 并 通过调节成膜分子的电荷密度、头基间距、膜的物理 状态等而使之与特定性质的细胞膜相似 $[9,10]$. 因此,
用磷脂 LB膜作为模板来研究草酸钙 $(\mathrm{CaOxa})$ 晶体的 形成过程, 比在水溶液中更能接近 $\mathrm{CaOxa}$ 结石在生物 体内尿路细胞膜表面形成的真实情况. 特别是利用 存在缺陷的 LB膜可以模拟受损伤的肾上皮细胞膜诱 导肾结石矿物 $\mathrm{CaOxa}$ 晶体生长, 为进一步探讨尿石 形成的机理、更有效地治疗尿路结石病和临床上寻找 修复损伤膜的药物提供重要的参考价值.

\section{1 实验部分}

\section{1 仪器与试剂}

二棕榈酰磷脂酰胆碱(DPPC)为 Sigma 公司产品; 荧光探针分子 1-十六烷酰-2-[12-[(7-硝基-2-1,3-苯基 口恶二唑-4-基)氨基]十二烷酰]-sn-丙三氧基-3-胆碱磷 酸(NBD-PC)购自 Avanti Polar Lipids (Alabaster, AL); 草酸钾、氯化钙、氯化钠和氯仿等均为分析纯试剂; Tris. HCl 试剂由三羟甲基甲胺和盐酸配制而成, 原始 $\mathrm{pH}=2.04$, 用 $\mathrm{NaOH}$ 溶液调节 $\mathrm{pH}$. 实验用水均为从石 英亚沸蒸馏器体系制得的二次蒸馏水; 孔径 $0.22 \mu \mathrm{m}$ 
的微孔滤膜为上海半岛实业有限公司净化器材厂生 产.

XL-30 型环境扫描电子显微镜(ESEM, Philips 公 司), 样品喷金处理, 测量电压 $15 \sim 20 \mathrm{kV}$. KSV-3000 全自动 LB 膜仪(芬兰). PHS-3C 精密 $\mathrm{pH}$ 计(上海雷磁 仪器厂). 苂光显微镜为德国产品(Leica DMRA2), 苂 光标记物 NBD-PC 的浓度为 $1 \%$, 激发波长 $460 \mathrm{~nm}$, 发射波长 $534 \mathrm{~nm}$.

\section{2 实验方法}

单分子膜和LB膜的制备参照文献 [11,12]进行: 以二次水为亚相, 在 $L B$ 槽内铺展浓度为 $1.0 \mathrm{mmol} / \mathrm{L}$ 的磷脂DPPC氯仿溶液, 待水面上氯仿挥发 $20 \mathrm{~min}$ 后, 以 $0.03 \mathrm{~nm}^{2} \cdot \mathrm{molecul}^{-1} \cdot \mathrm{min}^{-1}$ 的速度压膜至目标表面 压，采用垂直提拉法将单分子膜转移到云母基片上， 提膜速度为 $3 \mathrm{~mm} / \mathrm{min}$, 沉积表面压 $10 \mathrm{mN} / \mathrm{m}$. DPPC 亲水功能基团朝外, 单层膜, 在干燥器中放置 $1 \mathrm{~d}$ 后, 按照下面方法制备缺陷LB膜.

缺陷 LB 膜制备: 将上述方法制备的 LB 膜浸泡 在 $0.6 \mathrm{mmol} / \mathrm{L}$ 的草酸钾溶液中, 浸泡时间为 $2 \mathrm{~h}$. 取 出后用二次水小心洗去膜表面的草酸钾，干燥，将其 中一片进行 AFM 观察, 另一片放入 $\mathrm{CaOxa}$ 亚稳溶液 中进行晶体生长.
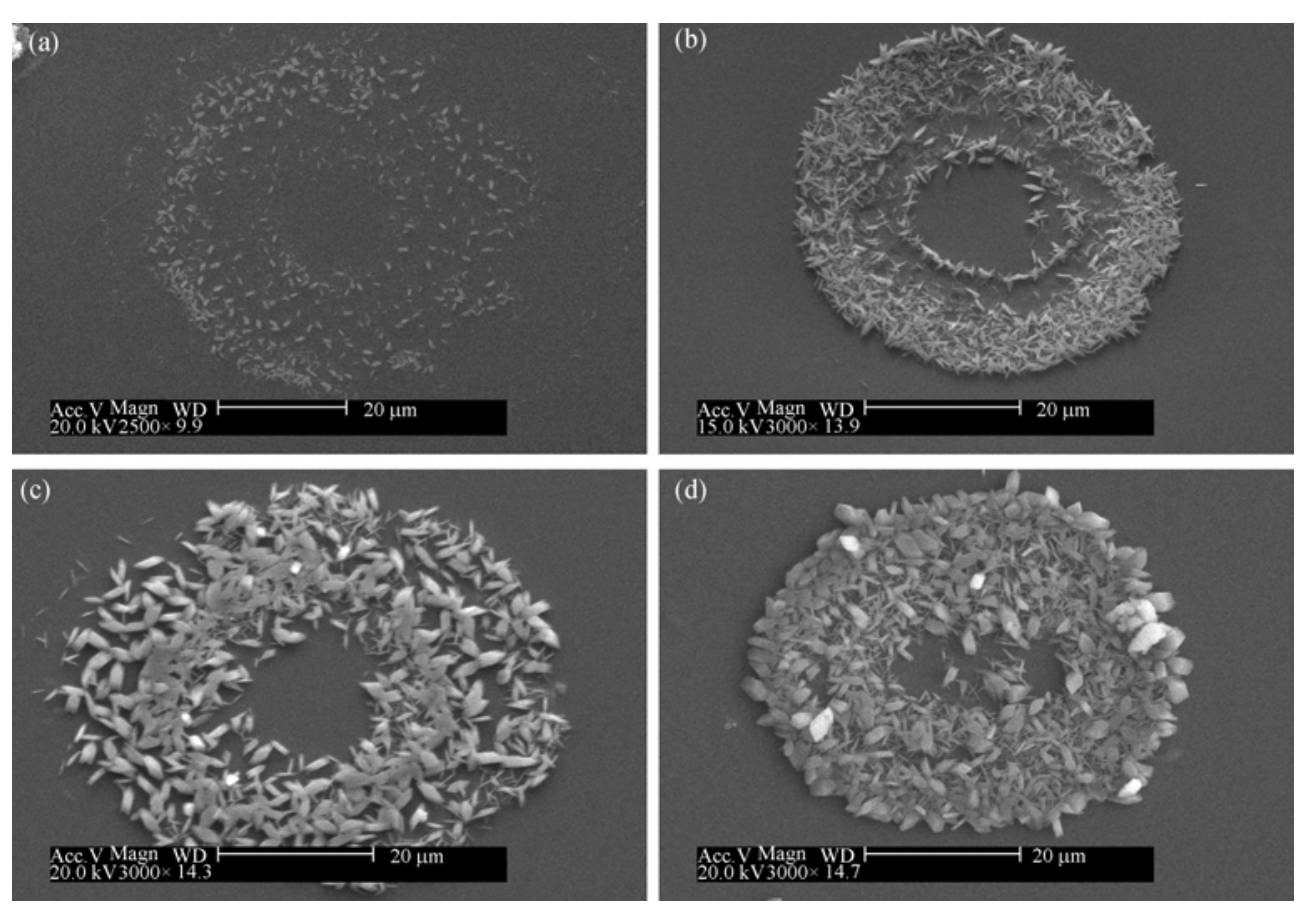

图 1 在不同时间后, 缺陷 LB 膜诱导 $\mathrm{CaOxa}$ 亚稳溶液生成的环状 COM 晶体图形的 SEM 照片

(a) $20 \mathrm{~min}$; (b) $1 \mathrm{~h}$; (c) $4 \mathrm{~h}$; (d) $24 \mathrm{~h}$. LB 膜沉积表面压: $10 \mathrm{mN} / \mathrm{m}$; $c(\mathrm{CaOxa})=0.30 \mathrm{mmol} \cdot \mathrm{L}^{-1}$; 云母基片; 晶体生长温度: (30 \pm 1$)^{\circ} \mathrm{C}$ 用孔径为 $0.22 \mu \mathrm{m}$ 的微孔滤膜过滤的CaOxa亚稳溶 液, 最终浓度 $c\left(\mathrm{Ca}^{2+}\right)=c\left(\mathrm{Oxa}^{2-}\right)=0.30 \mathrm{mmol} / \mathrm{L}, c(\mathrm{NaCl})=$ $10 \mathrm{mmol} / \mathrm{L}$. 然后将制备好的LB膜水平放置在气液界 面处, 有膜一面朝向 $\mathrm{CaOxa}$ 溶液. 晶体生长在密闭环 境中进行, 以防止由于溶剂水的挥发而造成体系饱 和度的变化. 整个实验在无尘箱内完成, 重复 3 次, 温度为 $(30 \pm 1)^{\circ} \mathrm{C}$.

\section{2 结果与讨论}

\section{1 缺陷 LB 膜诱导 COM 晶体的形貌}

图 1 为被草酸钾损伤后的缺陷 LB 膜诱导一水草 酸钙(COM)生长不同时间后晶体的 SEM 照片. 当在 $0.3 \mathrm{mmol} / \mathrm{L}$ 的 $\mathrm{CaOxa}$ 亚稳溶液中生长 $20 \mathrm{~min}$ 后, 就 可在缺陷 LB 膜上发现由 COM 微晶排列成的环状晶 体图形(图 1(a)), 在环外的 COM 晶体很少, 但此时环 状图形的边界不太清晰. 构成环状图形的 COM 晶体 很薄, 尺寸约 1 2 $\mu \mathrm{m}$, 大都是针状或由针状晶体发 展而成的棱角不很清晰的六边形、棱形和叉生 COM 晶体. 相比之下, 没有被草酸钾处理的 DPPC LB 膜 在 $1 \mathrm{~h}$ 后才可在其表面生长出相当于图 1(a)尺寸(1 2 $\mu \mathrm{m})$ 的 COM 微晶; 而没有 LB 膜存在时, 至少 $6 \mathrm{~h}$ 才 
有 CaOxa 均相沉淀产生. 即促进 COM 晶体的异相成 核的强度依次为: 缺陷 LB 膜 $>$ 常规 LB 膜 $>$ 无 LB 膜水溶液.

随着生长时间增加到 $40 \mathrm{~min} 、 1 \mathrm{~h}$ (图 1(b))、2 h 和 $4 \mathrm{~h}$ (图 1(c))后, 单个晶体的尺寸逐渐增加, 针状晶 体减少，出现更多叉生和棱形的 COM 晶体，同时环 状图案里面 $\mathrm{COM}$ 晶体数量也增加. 与生长时间为 20 $\min$ 相比, 生长 $4 \mathrm{~h}$ 的 COM 晶体(图 1(c))尺寸增大至 $4 \sim 5 \mu \mathrm{m}$. 至生长 $24 \mathrm{~h}$ 后, 环内基本填满了 $\mathrm{COM}$ 晶体, 但环外晶体仍然较少.

相比之下，未经草酸钾处理的LB膜则不能诱导 生成环状 $\mathrm{COM}$ 晶体图形, 而是诱导生成单个的六边 形COM晶体(图 2), 零散地分散在LB膜表面; 生长 24 $\mathrm{h}$ 后其尺寸增加至 $10 \mu \mathrm{m}$ 左右，远大于相同条件下缺 陷LB膜诱导的COM微晶的尺寸(图 1(d)). Khan等 [14,15] 的研究也表明, 在磷脂单分子膜表面诱导生成的 $\mathrm{COM}$ 晶体主要为六边形.

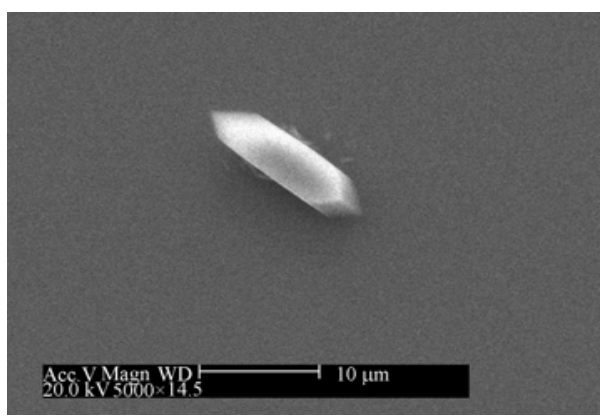

图 2 未经草酸钾处理的 LB 膜诱导的草酸钙晶体的 SEM 照片

在饱和溶液中，晶体有两种可能的成核方式：一 种是在溶液中发生均相成核; 另一种则是在已有物 质表面发生异相成核. LB膜诱导晶体成核实际上就 是促进异相成核而抑制均相成核 [16]. 缺陷LB膜在很 短的时间内就能诱导生成由大量COM微晶聚集而成 的环状图形，说明缺陷LB膜比一般常规LB膜更能促 进异相成核. 缺陷LB膜诱导晶体图形的出现是由于 缺陷畴区尤其是缺陷的边界能提供更多的成核位点. 图 3 为含 $1 \%$ 苂光探针分子NBD-PC的DPPC缺陷LB膜 的荧光显微镜图片，可以清楚地看到尺寸约 10 20 $\mu \mathrm{m}$ 的畴区结构. Talham ${ }^{[2,11]}$, Vanderlick ${ }^{[17]}$ 和Kane $\mathrm{e}^{[18]}$ 等 的研究也证明, DPPC单分子膜存在圆形的缺陷畴区.
我们的结果不但反过来证明了DPPC单分子膜上的确 存在圆形缺陷，而且，这些缺陷畴区可优先促进 $\mathrm{COM}$ 晶体的成核.

Vogel等 ${ }^{[19]}$ 的研究表明, DPPC单分子膜诱导下的

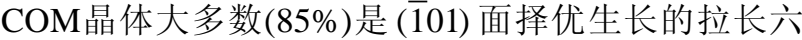
边形(图 4(a)), 少量(15\%)为(010)面择优生长的拉长 方块形(图 4(b)). 而环状图形里面的 COM晶体则还包 括更多的形貌，如叉生、针状和棱形COM晶体，这种 情况与在各种水溶液体系 [20]和在凝胶体系 ${ }^{[21]}$ 中常见 的COM晶体形貌更接近。这就表明，缺陷LB膜在诱 导 $\mathrm{COM}$ 晶体的生长过程中, 缺陷主要起到提供成核

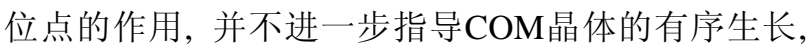
因为在这些诱导 COM成核的缺陷处，DPPC分子排列 无序，难以对 $\mathrm{COM}$ 晶体的有序生长起指导作用，导 致环状图形里面 $\mathrm{COM}$ 晶体的形貌与在水溶液和凝胶 体系中形成的 COM 晶体相似. 而分子有序排列的常 规LB膜却能在诱导 COM成核后，继续指导 $\mathrm{COM}$ 晶体 的整个生长过程，使得 $\mathrm{COM}$ 晶体沿一定晶面择优生 长, 最终得到规则的具有立体感的拉长六边形 $\mathrm{COM}$ 晶体 $[12,22,23]$. 这也从另一方面证明了环状 COM晶体 图形是由于LB膜的缺陷诱导而成.

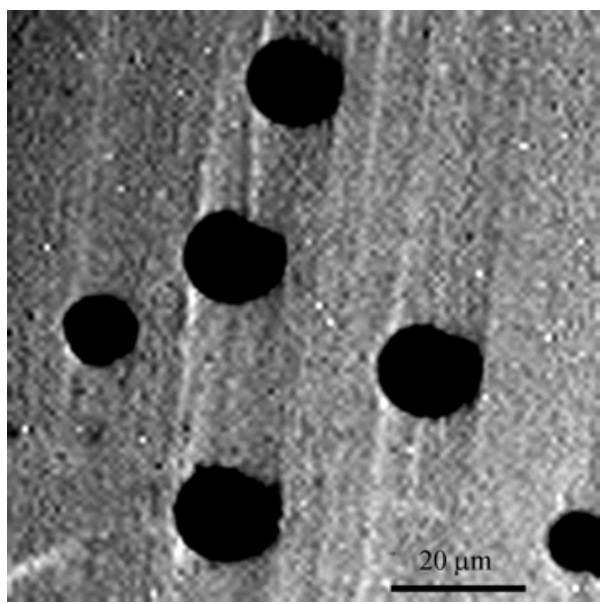

图 3 缺陷 LB 膜 LC/LE 畴区的苂光显微镜图像

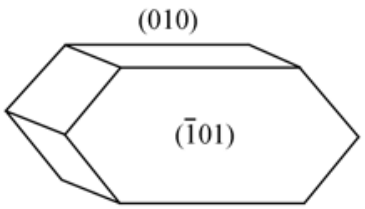

(a)

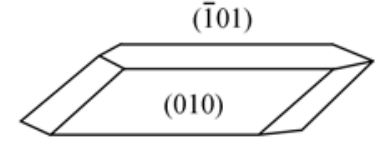

(b)
图 4 不同形貌COM晶体示意图 [17] 


\section{2 缺陷 LB 膜诱导的 COM 晶体图形尺寸分布}

环状晶体图形的尺寸可分为大 $(50 \sim 200 \mu \mathrm{m})$ 、中 (20 50 $\mu \mathrm{m})$ 和小 $(5 \sim 20 \mu \mathrm{m})$ 三类. 通过对相同基片面 积(约 $40 \mathrm{~mm}^{2}$ )内的环状晶体图形进行统计, 基片上环 状晶体图形的数目从 49 个到 148 个不等, 且膜上图 形的分布不是很均匀. 随着生长时间的增加, 小尺寸 的环状晶体图形数量增加, 从 $20 \mathrm{~min}$ 的 $20.4 \%$, 增加 到 $1 \mathrm{~h}$ 的 $40.6 \%, 4 \mathrm{~h}$ 的 $62.5 \%$ 和 $24 \mathrm{~h}$ 的 $81.7 \%$ (图 5); 大 尺寸的环状晶体图形数量从 $20 \mathrm{~min}$ 的 $51.0 \%$, 下降到 $1 \mathrm{~h}$ 的 $23.4 \%, 4 \mathrm{~h}$ 的 $7.9 \%$ 和 $24 \mathrm{~h}$ 的 $4.8 \%$; 中等尺寸的 环状晶体图形的百分比在生长 $20 \mathrm{~min}, 1 \mathrm{~h}$ 和 $4 \mathrm{~h}$ 后分 别为 30.6\%, 35.9\%和 29.7\%. 图 6 为在不同生长时间 内缺陷 LB 膜诱导的不同尺寸 COM 晶体环图形的 SEM 照片, 可以看到, 随着生长时间增加, 小尺寸环 状晶体图形数量增加.

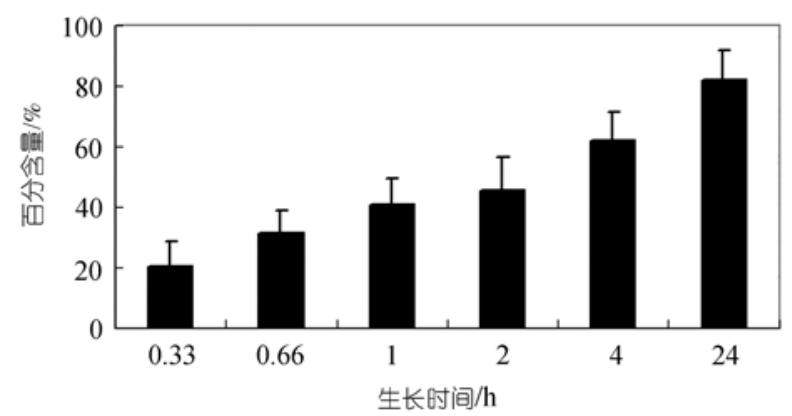

图 5 在不同生长时间内缺陷 LB 膜诱导的小尺寸环状晶 体图形的比例

上述结果是因为在 $L B$ 膜内, 大尺寸的缺陷畴区 边界不但缺陷位点多, 能提供更多的成核位点, 而且 其DPPC分子列阵比小尺寸缺陷畴区边界的分子更加 无序, COM初始微晶在此处的成核自由能更低 ${ }^{[22]}$, 因此, 最先诱导出与缺陷本身形貌相似的大尺寸环 状COM微晶图形生成, 即在生长时间比较短(如 20 $\mathrm{min}$ )时主要诱导大尺寸的环状晶体图形(占 51\%). 随 着生长时间的延长, 这些大尺寸缺陷畴区被 $\mathrm{COM}$ 微
晶占据后, COM便开始在尺寸越来越小的环状缺陷 处成核和生长，导致小尺寸的环状晶体图形的比例 逐渐增加.

中等尺寸 $(20 \sim 50 \mu \mathrm{m})$ 晶体环的比例, 在生长 20 $\min$ 至 $4 \mathrm{~h}$ 的期间内保持在 30\% 35\%左右，没有显著 的变化, 这归因于随着生长时间的增加, 在生长初期 诱导形成的小尺寸晶体图形会向环的两侧延伸生长, 导致环状晶体图形的半径变大, 如图 7 箭头 $\mathrm{A}$ 和 $\mathrm{B}$ 所示, 可以看出, 在晶体环的内侧和外侧, 主要为处 于生长初期的针状 COM 晶体, 其尺寸比环中间的晶 体尺寸小, 这说明晶体环的内侧和外侧均是晶体成 核和生长的活性部位. 因此, 生长时间延长后, 虽然 小尺寸的环形晶体数量增加使得中等尺寸的晶体图 形比例减小，但部分小尺寸的图形长大后又增加了 中等尺寸图形的数量, 最终其百分比变化不大.

随着生长时间的增加，不同尺寸的实心圆晶体 图案比例均增加. 小尺寸 $(5 \sim 20 \mu \mathrm{m})$ 的晶体环变成实 心圆晶体图案的时间最短, 生长 $1 \mathrm{~h}$ 后实心圆图案占 40.3\%, $2 \mathrm{~h}$ 后所有的小尺寸环状晶体图形里面基本上 均长满了 $\mathrm{COM}$ 晶体. 中等尺寸 $(20 \sim 50 \mu \mathrm{m})$ 的实心圆 晶体图案比例在生长 $1 \mathrm{~h}$ 后占 6.5\%, $2 \mathrm{~h}$ 后则上升到 $78.1 \%$. 而对于大尺寸 $(>50 \mu \mathrm{m})$ 的晶体图形, 在生长 $1 \mathrm{~h}$ 后仍然见不到实心圆图案, 在生长 $4 \mathrm{~h}$ 后, 环状图 形和实心圆图案约为 $1: 1$. 这些结果的产生归因于随 着生长时间的增加, 环状晶体图形从边界向内、外两 侧生长(图 7), 由于小尺寸的环容易长满晶体, 因此, 生长时间较短即可形成实心圆图形，而大尺寸的晶 体环即使在生长 $4 \mathrm{~h}$ 后仍然不能被 COM 全部覆盖.

\section{3 结论}

经过草酸钾处理后的 LB 膜由于存在圆形缺陷, 因此诱导生成了环形和圆形 COM 晶体图形, 相比之 下, 没有经草酸钾处理的 LB 膜只诱导零散的六边形 $\mathrm{COM}$ 晶体生成, 即促进 COM 晶体的异相成核的强度
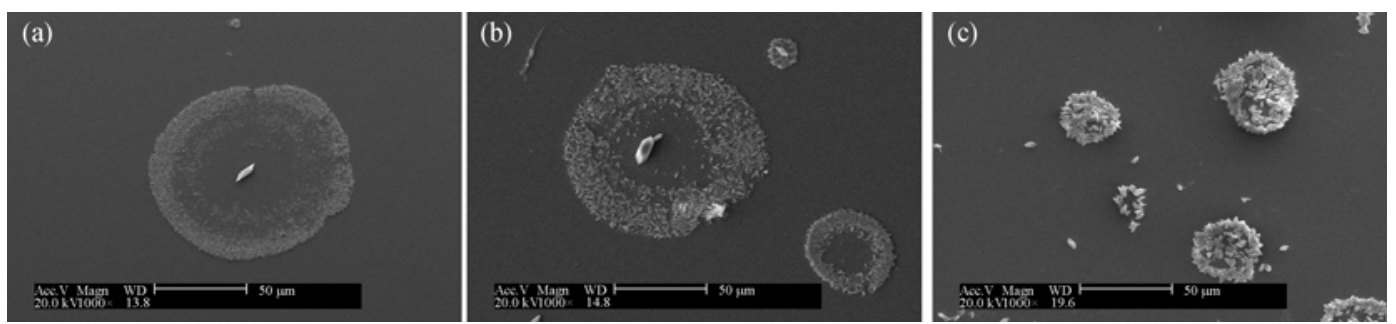

图 6 在不同生长时间内缺陷 LB 膜诱导的不同尺寸 COM 晶体环图形的 SEM 照片 (a) $1 \mathrm{~h}$; (b) $4 \mathrm{~h}$; (c) $24 \mathrm{~h}$ 


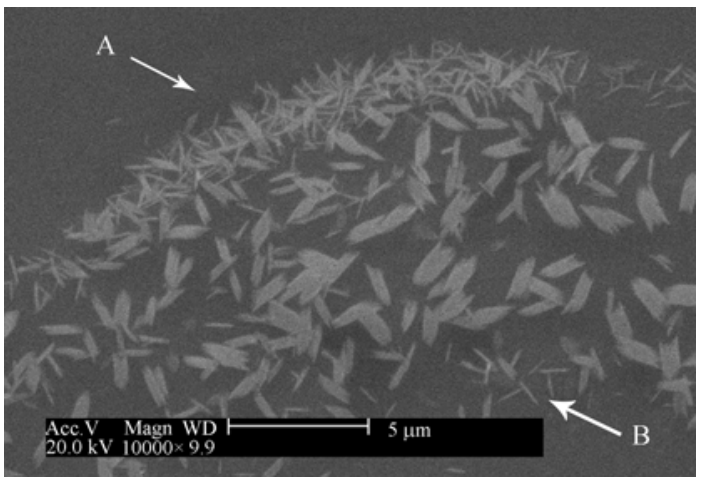

图 7 缺陷 $\mathrm{LB}$ 膜诱导生长 $20 \mathrm{~min}$ 后生长的 $\mathrm{COM}$ 晶体 SEM 的放大图

依次为: 缺陷 LB 膜 > 常规 LB 膜 > 无 LB 膜水溶 液. 这表明, 模型膜的缺陷是晶体生长的主要促进因 素. 随着生长时间增加, 环内单个 COM 晶体的尺寸 明显增加, 空心的环状 COM 晶体图形逐渐转变为实 心圆晶体图案. 由于 LB 膜的缺陷结构及其性质与受 损伤的肾上皮细胞膜表面具有相似性，因此，本文的 结果有助于从分子和超分子的水平上进一步了解肾 上皮细胞膜表面损伤后引起肾结石形成的分子机制.

\section{参考文献}

1 Wiessner J H, Hung L Y, Mandel N S. Crystal attachment to injured renal collecting duct cells: influence of urine proteins and $\mathrm{pH}$. Kidney Int, 2003, 63: 1313-1320 [DOI]

2 Benýtez I O, Talham D R. Calcium oxalate monohydrate precipitation at membrane lipid rafts. J Am Chem Soc, 2005, 127: 28142815 [DOI]

3 Khan S R, Shevock P N, Hackett R L. Membrane associated crystallization of calcium oxalate in vitro. Calc Tiss Int, 1990, 46: $116-120$ [DOI]

4 邓穗平，欧阳健明. 受损伤肾小管细胞膜诱导草酸钻结石形成 的分子机制. 江西医学院学报, 2005, 45(5): 165-167

5 欧阳健明. LB 膜原理与应用. 广州: 暨南大学出版社, 1999. 243 $-253$

6 Takamoto D Y, Aydil E, Zasadzinski J A, Ivanova A T, Schwartz D $\mathrm{K}$, Yang T, Cremer P S. Stable ordering in Langmuir-Blodgett films. Science, 2001, 293: 1292-1295 [DOI]

7 Peltonen J P K, He P, Rosenholm J B. Order and defects of Langmuir-Blodgett films detected with the atomic force microscope. $\mathrm{J}$ Am Chem Soc, 1992, 114(20): 7637-7642 [DOI]
8 Schwartz D K. Langmuir-Blodgett film structure. Surf Sci Rep, 1997, 27: 241-334 [DOI]

9 欧阳健明. 单分子膜调控草酸钙晶体成核和生长的研究进展. 科学通报, 2004, 49(2): 107-114

10 欧阳健明. 单分子膜诱导生物矿物晶体生长中的晶格匹配和电 荷匹配. 化学进展, 2005, 17(5): 931-937

11 Benýtez I O, Talham D R. Brewster angle microscopy of calcium oxalate monohydrate precipitation at phospholipid monolayer phase boundaries. Langmuir, 2004, 20: 8287-8293 [DOI]

12 Ouyang J M, Deng S P, Zhong J P, Tieke B, Yu S H. Crystallization of calcium oxalate monohydrate at dipalmitoylphosphatidylcholine monolayers in the presence of chondroitin sulfate A. J Cryst Growth, 2004, 270: 646-654 [DOI]

13 钟㺵平, 欧阳健明, 邓穗平. LB 膜诱导下草酸钙晶体的生物矿 化研究. 无机化学学报, 2002, 18(12): 1253-1257

14 Backov R, Khan S R, Mingotaud C, Byer K, Lee C M, Talham D R. Precipitation of calcium oxalate monohydrate at phospholipid monolayers. J Am Soc Nephrol, 1999, 10: S359-S363 [DOI]

15 Khan S R, Glenton P A, Backov R, Talham D R. Presence of lipids in urine, crystals and stones: implications for the formation of kidney stones. Kidney Int, 2002, 62: 2062-2072 [DOI]

16 Bunker B C, Rieke P C, Tarasevich B J, Campbell A A, Fryxell G E, Graff G L, Song L, Liu J, Virden J W, Mcvay G L. Ceramic thin-film formation on functionalized interfaces through biomimetic processing. Science, 1994, 264: 48-55 [DOI]

17 McConlogue C W, Vanderlick T K. A close look at domain formation in DPPC monolayers. Langmuir, 1997, 13: 7158-7164 [DOI]

18 Kane S A, Compton M, Wilder N. Interactions determining the growth of chiral domains in phospholipid monolayers: experimental results and comparison with theory. Langmuir, 2000, 16: 8447 -8455 [DOI]

19 Letellier S R, Lochhead M, Vogel V. Oriented growth of calcium oxalate monohydrate crystals beneath phospholipid monolayers. Biochim Biophy Acta-Gen Subject, 1998, 1380: 31—45

20 欧阳健明, 姚秀琼, 钟㺵平, 谢瑜珊, 白钰, 广荔, 唐雯霞. 不 同模拟体系中草酸钻结晶的比较研究. 高等学校化学学报, 2002, 23(12): 2237-2239

21 郑辉, 李祥平, 欧阳健明. 过饱和度、反应物化学计量比及离子 强度对草酸钙晶体生长的影响. 无机化学学报, 2005, 21(9): $1375-1378$

22 Whipps S, Khan S R. Growth of calcium oxalate monohydrate at phospholipid Langmuir monolayers. J Cryst Growth, 1998, 192: 243-249 [DOI]

23 Deng S P, Ouyang J M. Effects of dipalmitoylphosphatidylcholine monolayers to the crystallization of calcium oxalate monohydrate from the solution containing chondroitin sulfate C. Coll Surf A, 2005, 257-258: 47-50 [DOI] 